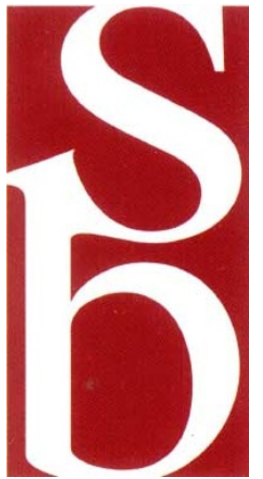

\title{
Aspirin and inflammation
}

Aspirin, paracetamol, ibuprofen, indomethacin and other non-steroidal anti-inflammatory drugs (NSAIDs) are important in the treatment of pain, fever, inflammation and allergy and represent a market worth $\$ 4.3$ billion. They exert their effects by interfering with the cyclooxygenase activity of the enzyme prostaglandin $\mathrm{H}_{2}$ synthetase (PGHS). In this issue of Nature Structural Biology, Michael Garavito and colleagues present the structure of PGHS-1 inactivated with an aspirin analogue, bromo-aspirin, and thereby detail the mechanism by which aspirin, the best known of the NSAIDs, works'.

The eicosanoids-prostaglandins, prostacyclins, thromboxanes and leukotrienes_-are signalling molecules involved in pain, fever and inflammatory responses, as well as muscle contraction and platelet aggregation. They are synthesized from arachidonic acid, a long-chain fatty acid derived from cellmembrane phospholipids: these synthetic pathways are the target for drugs such as the corticosteroid hormones, which block the synthesis of arachidonic acid, as well as the NSAIDs, which block the synthesis of prostaglandins, prostacyclins and thromboxanes.

The target for the NSAIDs, PGHS, is composed of three domains: a catalytic domain, a membrane-binding domain, and an epidermal growth factor (EGF) domain ${ }^{2}$. The catalytic domain is bifunctional, housing two separate active sites: the cyclooxygenase active site converts arachidonic acid to prostaglandin $\mathrm{G}_{2}$ which is then converted to prostaglandin $\mathrm{H}_{2}$ by the peroxidase active site. Prostaglandin $\mathrm{H}_{2}$ is released from PGHS and modified, in a cellspecific manner, into one of the several prostanoid hormones. PGHS is found on the luminal face of the endoplasmic reticulum membrane and is thought to function as a homodimer. There are two highly related forms of the protein: PGHS-l is constitutively ex- pressed in all tissues whereas expression of PGHS-2 is tissue specific and induced by inflammatory or mitogenic stimuli. It is this latter enzyme that is responsible for the rapid formation of prostanoid compounds during the inflammatory response.

The cyclooxygenase active site of PGHS is located at the end of a long tunnel, approximately $8 \times 25 \AA$, lined with hydrophobic residues that penetrates deep into the heart of each PGHS monomer; similar types of channels have been seen in other enzymes for which arachidonic acid is a substrate ${ }^{3}$. The mouth of the tunnel is formed by the membrane-binding domain which consists of four amphipathic helices whose hydrophobic residues face outwards, away from the protein. The membrane motifs in each monomer fall on the same side of the dimer, forming an extensive hydrophobic patch. The structure of these domains suggests that rather than interacting with the surface of the membrane or passing all the way through the lipid bilayer, they are likely to insert only halfway through the bilayer, three of the helices lying parallel to the plane of the membrane. If this model is correct, PGHS would be one of the monotopic class of membrane proteins.

Arachidonic acid is highly hydrophobic and, when released from the bilayer phospholipids by the action of phospholipases, is likely to remain associated with the membrane. The location of the protein, half buried in the bilayer, and the disposition of the tunnel entrance and its lining of hydrophobic residues provide a direct route from the centre of the membrane to the cyclooxygenase active site while sheltering the fatty-acid substrate from the surrounding solvent. An arachidonate molecule can be modelled into the active site, placing the $\mathrm{C}-13$ carbon in the vicinity of Tyr 385, which is known to be important in catalysis, and the carboxylate group next to Arg 120, one of only two nonhydrophobic residues found in the tunnel. 
Garavito and colleagues, in an effort to overcome the problems the low resolution of structure determinations often associated with membrane proteins, have synthesized a bromoderivative of aspirin. The bromo-aspirin is isosteric with aspirin and shows essentially the same activity against PGHS-1; the electrondense bromine atom allowed them to locate the compound unequivocally in the electron density map. The structure of the inactivated PGHS-1 reveals that, as expected, bromo-aspirin acetylates Ser 530 which lines the wall of the catalytic tunnel. Ser 530 is not involved in catalysis (its mutation to alanine has no effect on enzyme activity); rather, the dominant conformation of the acetylated residue is protruding into and plugging the tunnel directly below the Tyr 385 residue. Aspirin thus blocks access of the arachidonate to the upper portion of the tunnel and the active site. The NSAID Sflurbiprofen also blocks the active-site channel at the same position, although it does not covalently modify the enzyme ${ }^{2}$. Indeed, steric interference with substrate binding is thought to be the mode of action for all the NSAIDS, although only aspirin covalently modifies the enzyme.

The structure of the inactivated form of PGHS-1 may provide an explanation for the differing activity of aspirin against the two PGHS isoenzymes. The acetylated Ser 530 side chain seems to exist in two conformations; the major conformation blocks the catalytic tunnel whereas the minor conformation is folded back into an alcove leading off the channel. In this latter position the substrate could well squeeze past the acetylated residue and gain access to the active site. Garavito and colleagues speculate that the conformational preferences of the acetylated side chain may switched in the PGHS-2 enzyme, thereby nullifying the action of the drug. The altered conformation that the substrate may have to adopt to gain entrance to the modified active site could also explain why aspirin alters the product of the reaction catalysed by PGHS-2. Prostacyclin, produced by PGHS-1, is antithrombogenic when released by the endothelium and is cytoprotective when released by the gastric mucosa. Aspirin and indomethacin are much more active against PGHS-1 than PGHS-2 and this may be why these NSAIDs cause most damage to the stomach lining ${ }^{4}$.

The reaction of aspirin with PGHS produces the acetylated enzyme and sialic acid (SA) as the leaving group. Garavito and colleagues find additional density in the channel, directly below the modified Ser 530 residue, which they can assign to SA. Indeed, SA is known to be a competitive inhibitor of aspirin and the location of the SA in the channel suggests that it also acts to block access to the active site. The location of the SA also suggests that aspirin may initially bind in the same position, and then diffuse a further $5 \AA$ up the channel to reaction with Ser 530.

The fold of the catalytic domain of PGHS is very similar to that of other peroxidases, suggesting that they may have arisen from a common ancestor. But how did the cyclooxygenase tunnel and active site appear in the hydrophobic core of the peroxidase domain? It would seem that all that is required to open up the hydrophobic channel and active site is the shifting of an interior helix and substitution of a number of large residues for smaller ones. Addition of the four amphipathic helices of the membrane-binding domain would then complete the tunnel mouth.

The challenges remaining must be to determine the details of the interaction between PGHS and the lipid bilayer and to probe the function of the EGF domain. The structure of PGHS-2, which will surely appear soon, will provide a wealth of information for the drug designers to develop compounds highly selective for the PGHS-2 isoenzyme. The new generation of drugs will very likely render the present NSAIDs obsolete, although the role of aspirin as a preventive drug in heart attacks and strokes is likely to expand ${ }^{4}$.

1. Loll, P.J., Picot, D. \& Garavito, R.M. Nature struct. Biol. 2 637-643 (1995).

2. Picot, D., Loll, P.J. \& Garavito, R.M. Nature 367, 243-249 (1994).

3. Garavito, R.M., Picot, D. \& Loll,

P.J. Curr. Opin. struct. Biol. 4, 529-535 (1994).

4. Vane, J. Nature 367, 215-216

(1995).

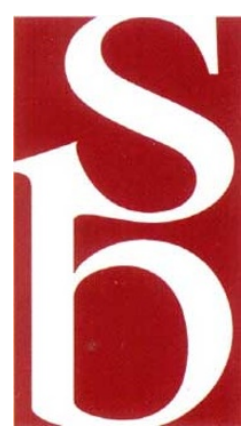

